\title{
Recently observed $\boldsymbol{P}_{c}$ as molecular states and possible mixture of $\boldsymbol{P}_{\boldsymbol{c}}(\mathbf{4 4 5 7})$
}

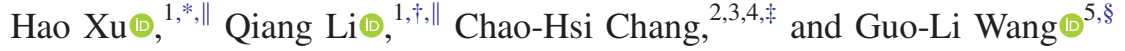 \\ ${ }^{1}$ School of Physical Science and Technology, Northwestern Polytechnical University, Xi' an 710129, China \\ ${ }^{2}$ Institute of Theoretical Physics, Chinese Academy of Sciences, Beijing 100190, China \\ ${ }^{3}$ School of Physical Sciences, University of Chinese Academy of Sciences, \\ 19A Yuquan Road, Beijing 100049, China \\ ${ }^{4}$ CCAST (World Laboratory), P.O. Box 8730, Beijing 100190, China \\ ${ }^{5}$ Department of Physics, Hebei University, Baoding 071002, China
}

(Received 15 January 2020; revised manuscript received 28 February 2020; accepted 9 March 2020; published 27 March 2020)

A recently observed spectrum of $P_{c}$ states exhibits a strong link to $\Sigma_{c} \bar{D}^{(*)}$ thresholds. In spite of successful molecular interpretations, we still push forward to wonder whether there exist finer structures. Utilizing the effecitve Lagrangians respecting heavy quark symmetry and chiral symmetry, as well as instantaneous Bethe-Salpeter equations, we investigate the $\Sigma_{c} \bar{D}^{(*)}$ interactions and three $P_{c}$ states. We confirm that $P_{c}(4312)$ and $P_{c}(4440)$ are good candidates of $\Sigma_{c} \bar{D}$ and $\Sigma_{c} \bar{D}^{*}$ molecules with spin- $\frac{1}{2}$, respectively. Unlike other molecular calculations, our results indicate the $P_{c}(4457)$ signal might be a mixture of spin- $\frac{3}{2}$ and spin- $-\frac{1}{2} \Sigma_{c} \bar{D}^{*}$ molecules, where the latter one appears to be an excitation of $P_{c}(4440)$. Therefore we conclude that, confronting three $\mathrm{LHCb} P_{c}$ signals, there may exist not three, but four molecular states.

DOI: 10.1103/PhysRevD.101.054037

\section{INTRODUCTION}

The study of exotic states, especially $X Y Z$ and pentaquarks, has became a hot topic in recent years. A benefit from upgraded $\tau$-charm and $b$ factories such BESIII, $\mathrm{LHCb}$, and Belle, a large amount of tetraquark candidates, as well as two pentaquark candidates were observed. These findings indeed extend our knowledge about nonperturbative QCD. However, the properties and inner structures of the states are still in debate; see Refs. [1-4] for reviews of experimental and theoretical status.

The great progress was made in 2015, when two pentaquark candidates $P_{c}(4380)$ and $P_{c}(4450)$ were reported by the LHCb Collaboration [5]. With an amplitude analysis, LHCb studied the process $\Lambda_{b} \rightarrow J / \psi K^{-} p$ and observed them in $J / \psi p$ final states. Both resonances have to be fulfilled with minimal quark content $c \bar{c} u u d$; therefore, they are good candidates of hidden-charm pentaquarks. Just

\footnotetext{
*xuh2018@nwpu.edu.cn

Corresponding author.

liruo@nwpu.edu.cn

*hangzx@itp.ac.cn

\$gl_wang@hit.edu.cn

These authors contribute equally to this work.
}

Published by the American Physical Society under the terms of the Creative Commons Attribution 4.0 International license. Further distribution of this work must maintain attribution to the author(s) and the published article's title, journal citation, and DOI. Funded by SCOAP ${ }^{3}$. after the discovery, different dynamics were applied to look into their natures: the molecular states of $\Sigma_{c} \bar{D}^{(*)}[6-10]$, the compact pentaquark structures [11-14], the dynamical effects [15-17], etc. Until now, people have made tremendous effects to clarify their constituents and quantum numbers (see Refs. [1,3] for reviews). Besides, some predictions were already made before the observations of the $P_{c}$ states [18-22].

Recently, LHCb [23] reexamined the process $\Lambda_{b} \rightarrow$ $J / \psi K^{-} p$ with nine times larger decay samples compared to Ref. [5], and revealed a more sophisticated structure in $J / \psi p$ invariant mass spectrum than before: the $P_{c}(4450)$ signal splits into two peaks $P_{c}(4440)$ and $P_{c}(4457)$, while a new pentaquark state $P_{c}(4312)$ shows up in the lower mass region. The parameters of these $P_{c}^{+}$states are collected in Table I. From Table I, we notice that the masses of $P_{c}(4440)$ and $P_{c}(4457)$ are slightly below $\Sigma_{c} \bar{D}^{*}$ threshold, while $P_{c}(4312)$ is quite close to $\Sigma_{c} \bar{D}$, therefore it is strongly believed that $\Sigma_{c} \bar{D}^{(*)}$ interactions are responsible for the enhancements in the $J / \psi p$ invariant spectrum. So far, a number of papers have come out to interpret the

TABLE I. Summary of the $P_{c}^{+}$properties observed by the LHCb.

\begin{tabular}{lcc}
\hline \hline State & $M[\mathrm{MeV}]$ & $\Gamma[\mathrm{MeV}]$ \\
\hline$P_{c}^{+}(4312)$ & $4311.9 \pm 0.7_{-0.6}^{+6.8}$ & $9.8 \pm 2.7_{-4.5}^{+3.7}$ \\
$P_{c}^{+}(4440)$ & $4440.3 \pm 1.3_{-4.7}^{+4.1}$ & $20.6 \pm 4.9_{-10.1}^{+8.7}$ \\
$P_{c}^{+}(4457)$ & $4457.3 \pm 0.6_{-1.7}^{+4.1}$ & $6.4 \pm 2.0_{-1.9}^{+5.7}$ \\
\hline \hline
\end{tabular}


new results, which carry different opinions such as the molecular states [24-45], pentaquarks [46-52], hadrocharmonium [53], etc.

As indicated above, $\Sigma_{c} \bar{D}^{(*)}$ molecular interpretations seem to be the most suitable option. Although many papers (such as Refs. [24,37]) have confirmed their molecular nature, it is still necessary to examine it from a different approach. Furthermore, the old $P_{c}(4450)$ splits into $P_{c}(4440)$ and $P_{c}(4457)$, so are there any chances that three $P_{c}$ states may have finer structures considering the complexity of threshold interaction?

To answer the question, we will study $\Sigma_{c} \bar{D}^{(*)}$ interactions and the three $P_{c}$ states. We first calculate the heavyhadron interaction amplitudes within the chiral symmetry and heavy quark symmetry [19,54-58], then iterate the obtained interaction kernel into the Bethe-Salpeter equation (BSE) to explore the nature of the $\Sigma_{c} \bar{D}^{(*)}$ heavy-hadron systems. The Bethe-Salpeter (BS) methods adopted here have been successfully applied to investigate the properties of the meson systems, including the mass spectra, hadronic transitions, and weak decays [59-67], as well as the recent $\Xi_{c c}$ study [68]. Therefore extending to the meson-baryon molecular systems is quite natural.

Besides, in the BS framework, the relativistic effects and the mixing of the different partial waves can be automatically involved, in spite of some approximations.

It is worth mentioning that, to understand the near threshold phenomenas and resonance formations in a two-hadron system, a nonperturbative resummation is quite crucial. Such resummation has been considered in chiral dynamics of nucleon-nucleon systems [69-71] and heavy meson systems [72], as well as the phenomenological studies of molecular states and $X Y Z$ exotics (see reviews $[1,2])$. A nonperturbative resummation is to partially summit interactions to all order, which can be achieved by a proper iterating equation such as the Lippmann-Schwinger equation, the Bethe-Salpeter equation, etc. However, the studies mentioned above take simplified or nonrelativistic equations. Therefore, in this article we also want to focus on the BS equation itself to push forward the resummation method.

In the present work, we will study $P_{c}(4312), P_{c}(4440)$, and $P_{c}(4457)$ states by investigating $\Sigma_{c} \bar{D}^{(*)}$ interactions. First, we adopt the effective Lagrangians with the heavy quark symmetry and chiral symmetry to calculate the transition amplitudes of the $\Sigma_{c} \bar{D}^{(*)}$ interactions. Then, we iterate them into the instantaneous Bethe-Salpeter equation, and look for the bound state solutions. With careful studies of the meson-baryon interactions, we hope that the natures of three $P_{c}$ states, which are astonishingly close to the corresponding thresholds, can be answered. One notices that there exists similar works with different approximations: Refs. [26,73] studied $P_{c}(4312)$, $P_{c}(4440)$, and $P_{c}(4457)$ with a quasipotential BetheSalpeter equation approach; Ref. [74] studied one of three $P_{c}$ states $P_{c}(4312)$ with the Bethe-Salpeter equation.
This work is organized as follows. After the Introduction, we introduce the effective Lagrangians, and present the calculated interaction kernels (Sec. II). In Sec. III, we exhibit the Bethe-Salpeter equations for $\Sigma_{c} \bar{D}^{(*)}$ interactions. In Sec. IV numerical results and some discussions are presented. Section V denotes to a brief summary and conclusion of this work.

\section{LAGRANGIANS AND $\boldsymbol{\Sigma}_{c} \overline{\boldsymbol{D}}^{(*)}$ INTERACTION KERNELS}

To study the $\Sigma_{c} \bar{D}^{(*)}$ interactions later, we illustrate the corresponding Lagrangians first. The interaction between an $S$-wave heavy-light meson and a light pseudoscalar meson reads [54-57]

$$
\mathcal{L}_{H_{c} P}=+g\left\langle\bar{H}_{c} \psi l \gamma_{5} H_{c}\right\rangle .
$$

In the above, the $H_{c}$ field represents the $\left(\bar{D}, \bar{D}^{*}\right)$ doublet in the heavy quark limit

$$
\begin{aligned}
H_{c} & =\left(P_{c \mu}^{*} \gamma^{\mu}+i P_{c} \gamma_{5}\right) \frac{1-\not \gamma}{2}, \\
\bar{H}_{c} & =\gamma^{0} H_{c}^{\dagger} \gamma^{0}=\frac{1-\not p}{2}\left(P_{c \mu}^{* \dagger} \gamma^{\mu}+i P_{c}^{\dagger} \gamma_{5}\right), \\
P_{c} & =\left(\bar{D}^{0}, D^{-}, D_{s}^{-}\right), P_{c \mu}^{*}=\left(\bar{D}^{* 0}, D^{*-}, D_{s}^{*-}\right)_{\mu} .
\end{aligned}
$$

$v=(1,0,0,0)$ stands for the 4 -velocity of the $H$ field. The axial vector field $u$ is expressed as $u_{\mu}=\frac{i}{2}\left\{\xi^{\dagger}, \partial_{\mu} \xi\right\}=$ $-\frac{\partial_{\mu} \phi}{2 f}+\ldots$, where $\xi=\exp (i \phi / 2 f), f$ is the $\pi$ decay constant $\left(f_{\text {exp }}=92 \mathrm{MeV}\right)$, and

$$
\phi=\sqrt{2}\left(\begin{array}{ccc}
\frac{1}{\sqrt{2}} \pi^{0}+\frac{\eta}{\sqrt{6}} & \pi^{+} & K^{+} \\
\pi^{-} & -\frac{1}{\sqrt{2}} \pi^{0}+\frac{\eta}{\sqrt{6}} & K^{0} \\
K^{-} & \bar{K}^{0} & -\frac{2 \eta}{\sqrt{6}}
\end{array}\right) .
$$

Similarly, the interactions between heavy-light and lightvector(-scalar) mesons read [19,57]

$$
\begin{aligned}
\mathcal{L}_{H V} & =i \beta\left\langle\bar{H}_{c} v_{\mu} V^{\mu} H_{c}\right\rangle+i \lambda\left\langle\bar{H}_{c} \sigma_{\mu \nu} F^{\mu \nu}(V) H_{c}\right\rangle, \\
\mathcal{L}_{H \sigma} & =g_{s}\left\langle\bar{H}_{c} \sigma H_{c}\right\rangle,
\end{aligned}
$$

where $F_{\mu \nu}(V)=\partial_{\mu} V_{\nu}-\partial_{\nu} V_{\mu}+\left[V_{\mu}, V_{\nu}\right]$ and

$$
V=\frac{i g_{V}}{\sqrt{2}}\left(\begin{array}{ccc}
\frac{\rho^{0}}{\sqrt{2}}+\frac{\omega}{\sqrt{2}} & \rho^{+} & K^{*+} \\
\rho^{-} & -\frac{\rho^{0}}{\sqrt{2}}+\frac{\omega}{\sqrt{2}} & K^{* 0} \\
K^{*-} & \bar{K}^{* 0} & \phi
\end{array}\right) .
$$

The interactions between $S$-wave heavy baryon and light mesons are [19,56,58] 


$$
\begin{aligned}
\mathcal{L}_{S}= & i \frac{3}{2} g_{1} \epsilon^{\mu \nu \lambda \kappa} v_{\kappa}\left\langle\overline{\mathcal{S}}_{\mu} u_{\nu} \mathcal{S}_{\lambda}\right\rangle+i \beta_{S}\left\langle\overline{\mathcal{S}}_{\mu} v_{\alpha}\left(-V^{\alpha}\right) \mathcal{S}^{\mu}\right\rangle \\
& +\lambda_{S}\left\langle\overline{\mathcal{S}}_{\mu} F^{\mu \nu}(V) \mathcal{S}_{\nu}\right\rangle+\ell_{S}\left\langle\overline{\mathcal{S}}_{\mu} \sigma \mathcal{S}^{\mu}\right\rangle .
\end{aligned}
$$

Here, $\mathcal{S}_{\mu}^{a b}$ is composed of Dirac spinor operators

$$
\begin{aligned}
& \mathcal{S}_{\mu}^{a b}=-\sqrt{\frac{1}{3}}\left(\gamma_{\mu}+v_{\mu}\right) \gamma^{5} \mathcal{B}_{6}^{a b}+\mathcal{B}_{6 \mu}^{* a b}, \\
& \overline{\mathcal{S}}_{\mu}^{a b}=\sqrt{\frac{1}{3}} \overline{\mathcal{B}}_{6}^{a b} \gamma^{5}\left(\gamma_{\mu}+v_{\mu}\right)+\overline{\mathcal{B}}_{6 \mu}^{* a b},
\end{aligned}
$$

with

$$
\mathcal{B}_{6}=\left(\begin{array}{ccc}
\Sigma_{c}^{++} & \frac{1}{\sqrt{2}} \Sigma_{c}^{+} & \frac{1}{\sqrt{2}} \Xi_{c}^{\prime+} \\
\frac{1}{\sqrt{2}} \Sigma_{c}^{+} & \Sigma_{c}^{0} & \frac{1}{\sqrt{2}} \Xi_{c}^{\prime 0} \\
\frac{1}{\sqrt{2}} \Xi_{c}^{\prime+} & \frac{1}{\sqrt{2}} \Xi_{c}^{\prime 0} & \Omega_{c}^{0}
\end{array}\right) .
$$

We consider two isospin channels $\left(I=\frac{1}{2}, \frac{3}{2}\right)$ of $\Sigma_{c} \bar{D}^{(*)}$ in our work, which contain eigenstates:

$$
\begin{aligned}
& \left|\frac{1}{2}, \frac{1}{2}\right\rangle=\sqrt{\frac{2}{3}}\left|\Sigma_{c}^{++} ; \bar{D}^{(*)-}\right\rangle-\sqrt{\frac{1}{3}}\left|\Sigma_{c}^{+} ; \bar{D}^{(*) 0}\right\rangle, \\
& \left|\frac{3}{2}, \frac{1}{2}\right\rangle=\sqrt{\frac{1}{3}}\left|\Sigma_{c}^{++} ; \bar{D}^{(*)-}\right\rangle+\sqrt{\frac{2}{3}}\left|\Sigma_{c}^{+} ; \bar{D}^{(*) 0}\right\rangle .
\end{aligned}
$$

With the preparations above, we are able to calculate the interaction kernels which will be iterated into the instantaneous Bethe-Salpeter equation later. These kernels represent tree-level one-meson-exchange diagrams, including $\sigma, \pi, \eta$, $\rho$, and $\omega$ exchanges.

The calculated interaction kernel for $\Sigma_{c} \bar{D}$ is expressed as

$$
K\left(s_{\perp}\right)=F^{2}\left(s_{\perp}^{2}\right)\left(V_{1}+V_{2} \delta_{\perp}\right)
$$

where $F\left(s_{\perp}^{2}\right)$ denotes the form factor for the interaction vertexes.

In the following, we specify the $g_{S}, \ell_{S}, g, g_{1}, \beta, \beta_{S}, \lambda$, and $\lambda_{S}$ [in Eqs. (1), (4), (5) and (7)] to $\sigma_{1}, \sigma_{2}, \pi_{1}, \pi_{2}, \rho_{1}^{\mathrm{V}}, \rho_{2}^{\mathrm{V}}, \rho_{1}^{\mathrm{T}}$, and $\rho_{2}^{\mathrm{T}}$ respectively, for convenience. For $I=\frac{1}{2}$, potentials $V_{1}$ and $V_{2}$ read

$$
\begin{aligned}
& V_{1}=\frac{2 \sigma_{1} \sigma_{2}}{E_{\sigma}^{2}}+\rho_{1}^{\mathrm{V}} \rho_{2}^{\mathrm{V}} g_{V}^{2}\left(\frac{1}{E_{\rho}^{2}}+\frac{1}{2 E_{\omega}^{2}}\right), \\
& V_{2}=\frac{1}{3} \rho_{1}^{\mathrm{V}} \rho_{2}^{\mathrm{T}} g_{V}^{2}\left(\frac{2}{E_{\rho}^{2}}-\frac{1}{E_{\omega}^{2}}\right) .
\end{aligned}
$$

For $I=\frac{3}{2}, V_{1}$ and $V_{2}$ are

$$
\begin{aligned}
& V_{1}=\frac{2 \sigma_{1} \sigma_{2}}{E_{\sigma}^{2}}+\frac{1}{2} \rho_{1}^{\mathrm{V}} \rho_{2}^{\mathrm{V}} g_{V}^{2}\left(\frac{1}{E_{\omega}^{2}}+\frac{1}{E_{\rho}^{2}}\right), \\
& V_{2}=-\frac{1}{3} \rho_{1}^{\mathrm{V}} \rho_{2}^{\mathrm{T}} g_{V}^{2}\left(\frac{1}{E_{\rho}^{2}}+\frac{1}{E_{\omega}^{2}}\right) .
\end{aligned}
$$

In the above, $E_{\phi}=\sqrt{\vec{s}^{2}+m_{\phi}}$ denotes the energy of the exchanged meson, with $\phi=\sigma, \eta, \rho$, or $\omega$. Notice that in the $\Sigma_{c} \bar{D}$ interaction, only $\sigma$ and $\rho(\omega)$ exchanges contribute.

The interaction kernel for $\Sigma_{c} \bar{D}^{*}$ is written by

$$
\begin{aligned}
K^{\alpha \beta}\left(s_{\perp}\right)= & F^{2}\left(s_{\perp}^{2}\right)\left[\kappa_{1} g^{\alpha \beta}+\kappa_{2} \delta_{\perp} g^{\alpha \beta}+\kappa_{3} \delta_{\perp}\left(\gamma^{\alpha} s_{\perp}^{\beta}-\gamma^{\beta} s_{\perp}^{\alpha}\right)\right. \\
& \left.+\kappa_{4} \gamma^{\alpha} \gamma^{\beta}\right] .
\end{aligned}
$$

For the isospin- $-\frac{1}{2}$ states, we have potentials

$$
\begin{aligned}
\kappa_{1}= & -\frac{2 \sigma_{1} \sigma_{2}}{E_{\sigma}^{2}}+\frac{\pi_{1} \pi_{2} \vec{s}^{2}}{f^{2}}\left(\frac{1}{E_{\pi}^{2}}-\frac{1}{6 E_{\eta}^{2}}\right) \\
& +\rho_{1}^{\mathrm{V}} \rho_{2}^{\mathrm{V}} g_{V}^{2}\left(\frac{1}{E_{\rho}^{2}}-\frac{1}{2 E_{\omega}^{2}}\right), \\
\kappa_{2}= & \frac{1}{3} \rho_{1}^{\mathrm{V}} \rho_{2}^{\mathrm{T}} g_{V}^{2}\left(\frac{1}{E_{\omega}^{2}}-\frac{2}{E_{\rho}^{2}}\right), \\
\kappa_{3}= & \frac{\pi_{1} \pi_{2} \vec{s}^{2}}{f^{2}}\left(\frac{1}{E_{\pi}^{2}}-\frac{1}{6 E_{\eta}^{2}}\right)+\frac{2}{3} \rho_{1}^{\mathrm{T}} \rho_{2}^{\mathrm{T}} g_{V}^{2}\left(\frac{2}{E_{\rho}^{2}}-\frac{1}{E_{\omega}^{2}}\right), \\
\kappa_{4}= & -\frac{\pi_{1} \pi_{2} \vec{s}^{2}}{f^{2}}\left(\frac{1}{E_{\pi}^{2}}-\frac{1}{6 E_{\eta}^{2}}\right) .
\end{aligned}
$$

For the isospin- $-\frac{3}{2}$ states,

$$
\begin{aligned}
\kappa_{1}= & -\frac{2 \sigma_{1} \sigma_{2}}{E_{\sigma}^{2}}-\frac{\pi_{1} \pi_{2} \vec{s}^{2}}{2 f^{2}}\left(\frac{1}{E_{\pi}^{2}}+\frac{1}{3 E_{\eta}^{2}}\right) \\
& -\frac{1}{2} \rho_{1}^{\mathrm{V}} \rho_{2}^{\mathrm{V}} g_{V}^{2}\left(\frac{1}{E_{\rho}^{2}}+\frac{1}{E_{\omega}^{2}}\right),
\end{aligned}
$$

$\kappa_{2}=\frac{1}{3} \rho_{1}^{\mathrm{V}} \rho_{2}^{\mathrm{T}} g_{V}^{2}\left(\frac{1}{E_{\omega}^{2}}+\frac{1}{E_{\rho}^{2}}\right)$,

$\kappa_{3}=-\frac{\pi_{1} \pi_{2} \vec{s}^{2}}{2 f^{2}}\left(\frac{1}{E_{\pi}^{2}}+\frac{1}{3 E_{\eta}^{2}}\right)-\frac{2}{3} \rho_{1}^{\mathrm{T}} \rho_{2}^{\mathrm{T}} g_{V}^{2}\left(\frac{1}{E_{\rho}^{2}}+\frac{1}{E_{\omega}^{2}}\right)$,

$\kappa_{4}=\frac{\pi_{1} \pi_{2} \vec{S}^{2}}{2 f^{2}}\left(\frac{1}{E_{\pi}^{2}}+\frac{1}{3 E_{\eta}^{2}}\right)$.

\section{THE BETHE-SALPETER EQUATIONS OF THE $\Sigma_{c} \overline{\boldsymbol{D}}^{(*)}$ SYSTEMS}

In this part, we further study the Bethe-Salpeter formalism of the meson-baryon system $\Sigma_{c} \bar{D}^{(*)}$. Considering 
$J^{P}=1^{-}$or $0^{-}$for $\bar{D}^{(*)}$ and $J^{P}=\frac{1}{2}^{+}$for $\Sigma_{c}$, the corresponding Bethe-Salpeter equations and BS wave functions can be obtained.

\section{A. $\boldsymbol{\Sigma}_{c}\left(\frac{1}{2}^{+}\right) \bar{D}^{*}\left(\mathbf{1}^{-}\right)$system}

The Bethe-Salpeter equation for a meson-baryon system is schematically depicted in Fig. 1, which is written by

$$
\begin{aligned}
\Gamma^{\alpha}(P, q, r)= & \int \frac{\mathrm{d}^{4} k}{(2 \pi)^{4}}(-i) K^{\alpha \beta}\left(P, k_{\perp}, q_{\perp}\right) \\
& \times\left[S\left(k_{2}\right) \Gamma^{\gamma}(P, k, r) D_{\beta \gamma}\left(k_{1}\right)\right]
\end{aligned}
$$

where $\Gamma(P, q, r)$ denotes the pentaquark (refered as $P_{c}$ below) vertex carrying total momentum $P$, inner relative momentum $q$, and spin state $r$. Here we have on-shell condition $P^{2}=M^{2}$, with $M$ the $P_{c}$ mass. The inner relative momenta $q$ and $k$ are defined as

$$
q=\alpha_{2} p_{1}-\alpha_{1} p_{2}, \quad k=\alpha_{2} k_{1}-\alpha_{1} k_{2},
$$

with $\alpha_{1(2)} \equiv \frac{M_{1(2)}}{M_{1}+M_{2}} . k_{1(2)}$ and $M_{1(2)}$ are the momentum and mass of the meson component (baryon component), respectively. $S\left(k_{2}\right)=i \frac{1}{h_{2}-M_{2}}$ is the free propagator of the baryon, while the propagator $D^{\alpha \beta}$ (for the $J^{P}=1^{-}$meson) reads

$D^{\alpha \beta}\left(p_{1}\right)=D\left(p_{1}\right) d^{\alpha \beta}\left(p_{1 \perp}\right), \quad d^{\alpha \beta}\left(p_{1 \perp}\right)=-g^{\alpha \beta}+\frac{p_{1 \perp}^{\alpha} p_{1 \perp}^{\beta}}{M_{1}^{2}}$,

where $D\left(p_{1}\right)=i \frac{1}{p_{1}^{2}-M_{1}^{2}+i \epsilon}, p_{1 \perp}=p_{1}-p_{1} \cdot v v$.

We define a four-dimensional BS wave function and a three-dimensional Salpeter wave function below:

$$
\begin{aligned}
\psi_{\beta}(P, q) & =S\left(p_{2}\right) \Gamma^{\gamma}(P, q, r) D_{\beta \gamma}\left(p_{1}\right), \\
\varphi_{\beta}\left(P, q_{\perp}\right) & \equiv-i \int \frac{\mathrm{d} q_{P}}{2 \pi} \psi_{\beta}(P, q),
\end{aligned}
$$

where $q_{P}=q \cdot v$ and $q_{\perp}=q-q_{P} v$.

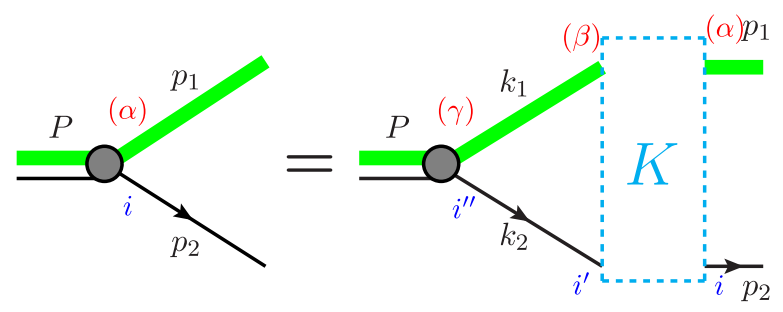

FIG. 1. The Bethe-Salpeter equation of the meson-baryon system. The Greeks (red) denote the Lorentz indices, while the Romans (blue) represent the Dirac indices. $P, p_{1}\left(k_{1}\right)$, and $p_{2}\left(k_{2}\right)$ stand for the momenta of the pentaquark, meson component, and the baryon component respectively.
Performing the contour integral over $q_{P}$ on both sides of Eq. (28) (see the Appendix for details), we obtain the Salpeter equation (SE),

$$
\begin{aligned}
\varphi_{\alpha}\left(P, q_{\perp}\right)= & \frac{1}{2 \omega_{1}}\left[\frac{\Lambda^{+}}{M-\omega_{1}-\omega_{2}}+\frac{\Lambda^{-}}{M+\omega_{1}+\omega_{2}}\right] d_{\alpha \beta}\left(p_{1 \perp}\right) \\
& \times \Gamma^{\beta}\left(P, q_{\perp}\right) .
\end{aligned}
$$

In the above, $\omega_{i}=\sqrt{M_{i}^{2}-p_{i \perp}^{2}}$ with $i=1,2$ stands for the kinematic energy of the constituent meson or baryon. We also have the projector operators,

$$
\Lambda^{ \pm}\left(p_{2 \perp}\right)=\frac{1}{2}\left[1 \pm \hat{H}\left(p_{2 \perp}\right)\right] \gamma^{0}
$$

with $\hat{H}=H / \omega_{2}$, and the Dirac Hamilton $H\left(p_{2 \perp}\right)=$ $\left(\not p_{2 \perp}+m_{2}\right) \gamma^{0}$.

We further split $\varphi$ into positive and negative energy wave functions:

$$
\varphi_{\beta}=\varphi_{\beta}^{+}+\varphi_{\beta}^{-}, \quad \varphi_{\beta}^{ \pm}\left(P, q_{\perp}\right)=\Lambda^{ \pm} \gamma^{0} \varphi_{\beta} .
$$

Notice in the weak binding condition $M \sim\left(\omega_{1}+\omega_{2}\right)$, we have $\varphi^{+} \gg \varphi^{-}$, i.e., the positive energy wave function $\varphi^{+}\left(q_{\perp}\right)$ dominates. Combined with Eq. (32), the SE of the $\Sigma_{c} \bar{D}^{*}$ system can be further simplified to a "Schrödingerlike" equation:

$$
M \varphi_{\alpha}=\left(\omega_{1}+\omega_{2}\right) \hat{H}\left(p_{2 \perp}\right) \varphi_{\alpha}+\frac{d_{\alpha \beta} \gamma^{0} \Gamma^{\beta}\left(q_{\perp}\right)}{2 \omega_{1}},
$$

with $\Gamma^{\beta}\left(q_{\perp}\right)$ the integral of the Salpeter wave function and the kernel,

$$
\Gamma^{\beta}\left(q_{\perp}\right)=\int \frac{\mathrm{d}^{3} k_{\perp}}{(2 \pi)^{3}} K^{\beta \gamma}\left(s_{\perp}\right) \varphi_{\gamma}\left(P, k_{\perp}\right) .
$$

Notice that the interaction kernel $K\left(s_{\perp}\right)$ is assumed to be instantaneous, thus has no dependence on the time component of the momentum transfer $s=(k-q)$.

Let us focus on the three-dimensional BSE (33). We can see that, the Salpeter wave function $\varphi_{\alpha}\left(q_{\perp}\right)$ in Eq. (30) is just transformed to an integral-type eigenvalue equation. In Eq. (33), the first term of the right side, which is determined by Dirac Hamiltonian $H$, stands for the kinetic energy. The second term contains the interaction kernel $K$, and therefore represents the potential energy.

In general, the normalization of a BS wave function is expressed as

$$
\begin{aligned}
& -i \iint \frac{\mathrm{d}^{4} q}{(2 \pi)^{4}} \frac{\mathrm{d}^{4} k}{(2 \pi)^{4}} \bar{\psi}_{\alpha}(P, q, \bar{r}) \frac{\partial}{\partial P^{0}} I^{\alpha \beta}(P, k, q) \psi_{\beta}(P, k, r) \\
& \quad=2 M \delta_{r \bar{r}},
\end{aligned}
$$


where

$$
\begin{aligned}
I^{\alpha \beta}(P, q, k)= & (2 \pi)^{2} \delta^{4}(k-q) S^{-1}\left(p_{2}\right) D^{-1 \alpha \beta}\left(p_{1}\right) \\
& +i K^{\alpha \beta}(P, k, q) .
\end{aligned}
$$

In the above, we have the inverse of the vector propagator

$$
D_{\alpha \beta}^{-1}\left(p_{1}\right)=\vartheta_{\alpha \beta} D^{-1}\left(p_{1}\right)
$$

with $\vartheta^{\alpha \beta}=-g^{\alpha \beta}+\frac{p_{1 \perp}^{\alpha} p_{1 \perp}^{\beta}}{\omega_{1}^{2}}$ as well as the identity $\vartheta^{\alpha \beta} d_{\beta \gamma}=\delta_{\gamma}^{\alpha}$.

As mentioned before, the interaction kernel is assumed to be no dependence on $P^{0}$ and $q_{P}$, namely, $K^{\alpha \beta}(P, k, q) \simeq$ $K^{\alpha \beta}\left(s_{\perp}\right)$; therefore the normalization would only involve the term related to two inverse propagators. After some deduction, Eq. (35) can be further simplified to

$$
\int \frac{\mathrm{d}^{3} q_{\perp}}{(2 \pi)^{3}} 2 \omega_{1} \vartheta^{\alpha \beta} \bar{\varphi}_{\alpha}\left(q_{\perp}, \bar{r}\right) \gamma^{0} \varphi_{\beta}\left(q_{\perp}, r\right)=2 M \delta_{r \bar{r}}
$$

which is just the normalization condition of the Salpeter equation (29).

\section{B. $\Sigma_{c}\left(\frac{1}{2}+\right) \bar{D}\left(0^{-}\right)$system}

Similarly, the Bethe-Salpeter equation for the $\Sigma_{c} \bar{D}$ system reads

$$
\begin{aligned}
\Gamma(P, q, r)= & \int \frac{\mathrm{d}^{4} k}{(2 \pi)^{4}}(-i) K\left(P, k_{\perp}, q_{\perp}\right) \\
& \times\left[S\left(k_{2}\right) \Gamma(P, k, r) D\left(k_{1}\right)\right],
\end{aligned}
$$

where $\Gamma(P, q, r)$ denotes the $P_{c}$ vertex. The BS wave function $\psi$ and related Salpeter wave function $\varphi$ are also defined as

$$
\begin{aligned}
\psi(P, q) & =S\left(k_{2}\right) \Gamma(P, q, r) D\left(q_{1}\right), \\
\varphi\left(P, q_{\perp}\right) & \equiv-i \int \frac{\mathrm{d} q_{P}}{2 \pi} \psi(P, q) .
\end{aligned}
$$

Performing the contour integral on $q_{P}$ over both sides of Eq. (39), we obtain the Salpeter equation

$$
M \varphi(q)=\left(\omega_{1}+\omega_{2}\right) \hat{H}\left(p_{2 \perp}\right) \varphi+\frac{\gamma^{0} \Gamma(q)}{2 \omega_{1}},
$$

with

$$
\Gamma\left(q_{\perp}\right)=\int \frac{\mathrm{d}^{3} k_{\perp}}{(2 \pi)^{3}} K\left(s_{\perp}\right) \varphi\left(P, k_{\perp}\right) .
$$

Applying the same strategy above, we obtain the normalization of $\varphi$ :

$$
\int \frac{\mathrm{d}^{3} q_{\perp}}{(2 \pi)^{3}} 2 \omega_{1} \bar{\varphi}\left(q_{\perp}, \bar{r}\right) \gamma^{0} \varphi\left(q_{\perp}, r\right)=2 M \delta_{r \bar{r}} .
$$

\section{The constructions of the Salpeter wave functions and further reductions}

We first turn to the $\Sigma_{c}\left(\frac{1}{2}^{+}\right) \bar{D}\left(0^{-}\right)$system. Accounting the spin-parity and the Lorentz structures, the Salpeter wave function $\left(J^{P}=\frac{1}{2}^{-}\right)$can be constructed as

$$
\begin{aligned}
\varphi\left(P, q_{\perp}, r\right) & =A\left(q_{\perp}\right) \gamma^{5} u(P, r) \\
& =\left(f_{1}+f_{2} \frac{q_{\perp}}{q}\right) \gamma^{5} u(P, r),
\end{aligned}
$$

where $f_{1(2)}(|\vec{q}|)$ only depends on $|\vec{q}|$.

It is worth mentioning that the wave function above can be rewritten in terms of the spherical harmonics $Y_{l}^{m}$ :

$$
\begin{aligned}
\varphi\left(P, q_{\perp}, r\right)= & C_{0}\left[f_{1} Y_{0}^{0}+C_{1} f_{2}\left(Y_{1}^{+1} \gamma^{-}+Y_{1}^{-1} \gamma^{+}-Y_{1}^{0} \gamma^{3}\right)\right] \\
& \times \gamma^{5} u(P, r),
\end{aligned}
$$

where $C_{0}=2 \sqrt{\pi}$ and $C_{1}=\frac{1}{\sqrt{3}} ; \quad \gamma^{ \pm}=\mp \frac{1}{\sqrt{2}}\left(\gamma^{1} \pm i \gamma^{2}\right)$. Therefore it is quite obvious that $f_{1}$ and $f_{2}$ represent $S$ - and $P$-wave components, respectively.

By inserting the wave function into Eq. (43), we obtain the normalization

$$
\int \frac{\mathrm{d}^{3} q_{\perp}}{(2 \pi)^{3}} 2 \omega_{1}\left(f_{1}^{2}+f_{2}^{2}\right)=1
$$

The $\frac{1-}{2}$ Salpeter wave function composed of $\Sigma_{c}\left(\frac{1}{2}^{+}\right) \bar{D}^{*}\left(1^{-}\right)$ can be written as

$$
\varphi_{\alpha}\left(P, q_{\perp}, r\right)=A_{\alpha}\left(q_{\perp}\right) u(P, r)
$$

with

$$
A_{\alpha}=\left(g_{1}+g_{2} \frac{q_{\perp}}{q}\right)\left(\gamma_{\alpha}-v_{\alpha}\right)+\left(g_{3}+g_{4} \frac{q_{\perp}}{q}\right) \hat{q}_{\perp \alpha},
$$

where $\hat{q}_{\perp \alpha}=\frac{q_{\perp \alpha}}{|\vec{q}|}, u(P, r)$ represents the Dirac spinor carrying momentum $P$ and spin state $r$. Notice that the radial wave function $g_{i}(|\vec{q}|)(i=1, \ldots, 4)$ only depends on $|\vec{q}|$. It is clear that $g_{1}$ corresponds to the $S$ wave, $g_{2(3)}$ belongs to the $P$ wave, and $g_{4}$ contributes both to $S$ and $D$ partial waves (see Ref. [68] for a further reading about different partial waves in terms of the spherical harmonics $Y_{l}^{m}$ ).

Inserting Eq. (47) into Eq. (37), we obtain the following normalization condition 


$$
\begin{aligned}
& \int \frac{\mathrm{d}^{3} q_{\perp}}{(2 \pi)^{3}} 2 \omega_{1}\left[3 c_{3}\left(g_{1}^{2}+g_{2}^{2}\right)+c_{1}\left(g_{3}^{2}+g_{4}^{2}-2 g_{1} g_{4}+2 g_{2} g_{3}\right)\right] \\
& \quad=1
\end{aligned}
$$

where $c=-q^{2} / \omega_{1}^{2}, c_{1}=1+c, c_{3}=1+c / 3$.

For the $\frac{3-}{2}$ state with $\Sigma_{c}\left(\frac{1}{2}^{+}\right) \bar{D}^{*}\left(1^{-}\right)$, the Salpeter wave function is written by

$$
\varphi_{\alpha}\left(P, q_{\perp}, r\right)=A_{\alpha \beta} \gamma^{5} u^{\beta}(P, r)
$$

where

$$
\begin{aligned}
A_{\alpha \beta}\left(q_{\perp}\right) \equiv & \left(h_{1}+h_{2} \frac{\phi_{\perp}}{q}\right) g_{\alpha \beta}+\left(h_{3}+h_{4} \frac{q_{\perp}}{q}\right)\left(\gamma_{\alpha}+v_{\alpha}\right) \hat{q}_{\perp \beta} \\
& +\left(h_{5}+h_{6} \frac{\phi_{\perp}}{q}\right) \hat{q}_{\perp \alpha} \hat{q}_{\perp \beta} .
\end{aligned}
$$

In Eq. (49), $u^{\beta}(P, r)$ is a Rarita-Schwinger spinor with polarization $r= \pm \frac{3}{2}, \pm \frac{1}{2}$. Note that the constructed Salpeter wave functions (47), (49) fulfills the condition $P^{\alpha} \varphi_{\alpha}=0$.

The normalization of Eq. (49) is calculated to be

$$
\begin{gathered}
\int \frac{\mathrm{d}^{3} q_{\perp}}{(2 \pi)^{3}} 2 \omega_{1}\left[c_{3} \sum_{i=1}^{4} h_{i}^{2}+\frac{1}{3} c_{1}\left(h_{5}^{2}+h_{6}^{2}+2 h_{4} h_{5}-2 h_{1} h_{5}\right.\right. \\
\left.\left.-2 h_{2} h_{6}-2 h_{3} h_{6}\right)+\frac{2}{3} c\left(h_{2} h_{3}-h_{1} h_{4}\right)\right]=1
\end{gathered}
$$

where the following completeness relation [75] has been used:

$$
\begin{aligned}
& \sum_{r} u^{\alpha}(P, r) \bar{u}^{\beta}(P, r) \\
& \quad=(P+M)\left[-g^{\alpha \beta}+\frac{1}{3} \gamma^{\alpha} \gamma^{\beta}-\frac{P^{\alpha} \gamma^{\beta}-P^{\beta} \gamma^{\alpha}}{3 M}+\frac{2 P^{\alpha} P^{\beta}}{3 M^{2}}\right] .
\end{aligned}
$$

With the above preparations, we are ready to transform Eqs. (33) and (41) into a set of coupled eigenvalue equations. For example, inserting the Salpeter wave function (44) into Eq. (41), we obtain

$$
\begin{aligned}
{[M} & \left.-\left(\omega_{1}+\omega_{2}\right) \hat{H}\left(p_{2 \perp}\right)\right] A\left(q_{\perp}\right) \gamma^{5} u(P, r) \\
& =\frac{1}{2 \omega_{1}} \gamma^{0} \int \frac{\mathrm{d}^{3} k_{\perp}}{(2 \pi)^{3}} K\left(s_{\perp}\right) A \gamma^{5} u(P, r) .
\end{aligned}
$$

After eliminating the spinor as well as projecting out the radial wave function, we obtain two coupled eigenvalue equations for the $\Sigma_{c} \bar{D}$ system

$$
\begin{aligned}
M f_{1}(|\vec{q}|)= & R_{11} f_{1}(|\vec{q}|)+R_{12} f_{2}(|\vec{q}|) \\
& -\frac{1}{2 \omega_{1}} \int \frac{\mathrm{d}^{3} \vec{k}}{(2 \pi)^{3}}\left[V_{1} f_{1}(|\vec{k}|)+\frac{\vec{s} \cdot \vec{k}}{k} V_{2} f_{2}(|\vec{k}|)\right],
\end{aligned}
$$

$$
\begin{aligned}
M f_{2}(|\vec{q}|)= & R_{21} f_{1}(|\vec{q}|)+R_{22} f_{2}(|\vec{q}|) \\
& -\frac{1}{2 \omega_{1}} \int \frac{\mathrm{d}^{3} \vec{k}}{(2 \pi)^{3}}\left[\frac{\vec{s} \cdot \vec{q}}{q} V_{2} f_{1}(|\vec{k}|)-\frac{\vec{k} \cdot \vec{q}}{k q} V_{1} f_{2}(|\vec{k}|)\right],
\end{aligned}
$$

where $\quad R_{22}=-R_{11}=m_{2}\left(\omega_{1}+\omega_{2}\right) / \omega_{2}, \quad R_{12}=R_{21}=$ $q\left(\omega_{1}+\omega_{2}\right) / \omega_{2}$. Here, the eigenvalue $M$ is just the $P_{c}$ mass. Solving these equations numerically, the corresponding mass spectra and wave functions can be obtained.

By inserting Eqs. (47) and (49) into Eq. (33), we can also work out the coupled eigenvalue equations for the $\Sigma_{c} \bar{D}^{*}$ system with $J^{P}=\frac{1}{2}^{-}, \frac{3}{2}^{-}$.

\section{NUMERICAL RESULTS AND DECODING THREE $\boldsymbol{P}_{\boldsymbol{c}}$ STATES}

In order to perform the numerical calculations, we first specify the values of the parameters used in this work $[6,19,24,26,76]$ :

$$
\begin{array}{llll}
\sigma_{1}=0.76, & \pi_{1}=0.59, & \rho_{1}^{\mathrm{V}}=0.9, & \rho_{1}^{\mathrm{T}}=0.56 \mathrm{GeV}^{-1}, \\
\sigma_{2}=6.2, & \pi_{2}=0.94, & \rho_{2}^{\mathrm{V}}=1.74, & \rho_{2}^{\mathrm{T}}=3.31 \mathrm{GeV}^{-1}, \\
g_{V}=5.9 . &
\end{array}
$$

We apply a monopole form factor in our work:

$$
F\left(s_{\perp}^{2}\right)=\frac{\Lambda^{2}}{-s_{\perp}^{2}+\Lambda^{2}},
$$

where $\Lambda$ is a parameter that characterizes the shape of the form factor, and usually set to the energy scale of the meson exchange. In our case, we fit it to the masses of $P_{c}(4312)$ and $P_{c}(4440)$, and we obtain $\Lambda=0.12 \mathrm{GeV}$ for $\Sigma_{c} \bar{D}^{*}$ and $0.18 \mathrm{GeV}$ for $\Sigma_{c} \bar{D}$. Notice that in the limit $s^{2} \rightarrow 0$, the heavy hadrons are treated as free and pointlike particles; therefore the form factor is normalized to 1 at $s^{2} \rightarrow 0$.

First, we illustrate the results of the potential $V_{i}$ and $\kappa_{i}$ with $I=\frac{1}{2}$ appearing in Eqs. (12) and (17). Their $s$ (transferred momentum) dependences are depicted in Fig. 2.

After solving the relevant eigenvalue equations, we find bound state solutions for $I=\frac{1}{2} \Sigma_{c} \bar{D}^{(*)}$ systems. The obtained mass spectra and corresponding binding energy are listed in Table II. To see the sensitivities of the calculations, we also vary $\Lambda$ by $\pm 5 \%$ as uncertainties.

For $\Sigma_{c} \bar{D}$, with the reasonable parameter $\Lambda$ the mass of experimental $P_{c}(4312)$ can be well reproduced, i.e., we obtain the meson-baryon bound state with mass $4.313 \mathrm{GeV}$. Therefore $P_{c}(4312)$ is a good candidate of the $I=\frac{1}{2} \Sigma_{c} \bar{D}$ molecular state carrying $J^{P}=\frac{1}{2}$. 


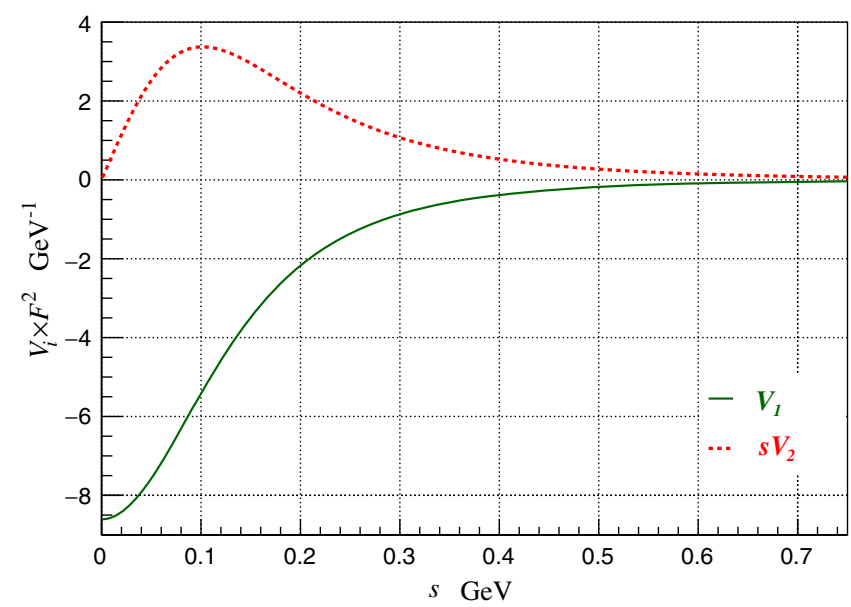

(a) $V_{i} \cdot F^{2}(i=1,2)$

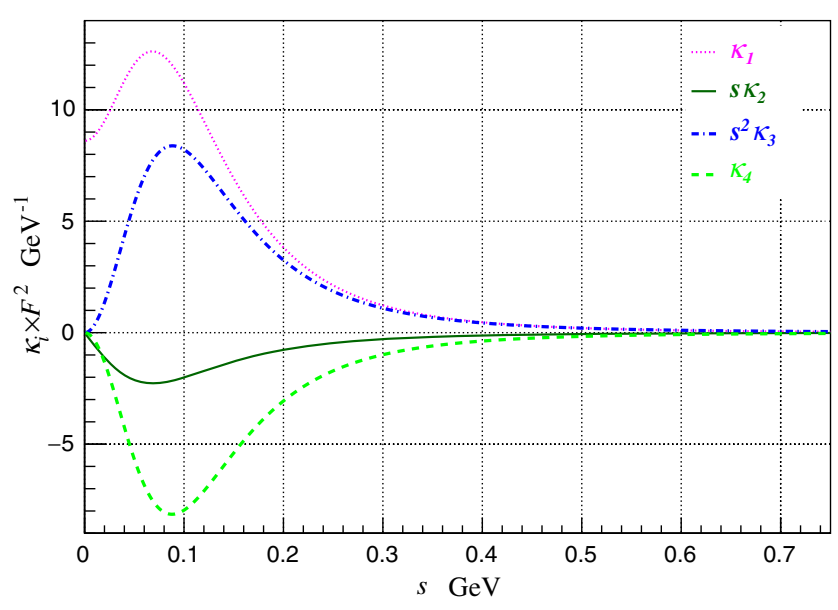

(b) $\kappa_{i} \cdot F^{2}(i=1, \cdots, 4)$

FIG. 2. The isospin- $\frac{1}{2}$ potentials $V_{i} \cdot F^{2}(i=1,2)$ and $\kappa_{n}$. $F^{2}(n=1, \ldots, 4)$ for $\Sigma \bar{D}$ and $\Sigma_{c} \bar{D}^{*}$, respectively.

For the $\Sigma_{c} \bar{D}^{*}$ system, we obtain three bound states: spin$\frac{1}{2}$ with mass $4.440 \mathrm{GeV}$ and spin $-\frac{1}{2}, \frac{3}{2}$ with $4.457 \mathrm{GeV}$. These three are well consistent with the experimental $P_{c}(4440)$ and $P_{c}(4457)$, respectively. We conclude $P_{c}(4440)$ can be treated as $I=\frac{1}{2} \Sigma_{c} \bar{D}^{*}$ molecular state carrying $J^{P}=\frac{1-}{2}$, while $P_{c}(4457)$ is $I=\frac{1}{2} \Sigma_{c} \bar{D}^{*}$ molecular state carrying $J^{P}=\frac{3}{2}^{-}$or $J^{P}=\frac{1}{2}^{-}$.

It is worth mention that, although two solutions of $\Sigma_{c} \bar{D}^{(*)}$ are fitted to $P_{c}(4312)$ and $P_{c}(4440)$, we still believe their

TABLE II. Calculated mass spectrum and binding energy $\Delta E$ (in $\mathrm{MeV}$ ) of the $\Sigma_{c} \bar{D}^{(*)}$ system with $I=\frac{1}{2}$, as well as categorized $P_{c}$.

\begin{tabular}{llrc}
\hline \hline$\Sigma_{c} \bar{D}^{(*)}\left(J^{P}\right)$ & \multicolumn{1}{c}{$M$} & $\Delta E$ & $P_{c}$ \\
\hline$\Sigma_{c} \bar{D}\left(\frac{1}{2}-\right)$ & $4313_{+2}^{-2}($ fitted $)$ & $-5_{-2}^{+2}$ & $P_{c}(4312)$ \\
$\Sigma_{c} \bar{D}^{*}\left(\frac{1}{2}-\right)$ & $4440_{+4}^{-5}($ fitted $)$ & $-20_{-4}^{+5}$ & $P_{c}(4440)$ \\
$\Sigma_{c} \bar{D}^{*}\left(\frac{3}{2}-\right)$ & $4457_{+1}^{-2}$ & $-3_{-1}^{+2}$ & $P_{c}(4457)$ \\
$\Sigma_{c} \bar{D}^{*}\left(\frac{1}{2}-\right)$ & $4456_{+1}^{-1}$ & $-4_{-1}^{+2}$ & $P_{c}(4457)$ \\
\hline \hline
\end{tabular}

$\Sigma_{c} \bar{D}^{(*)}$ explanations are preferred because of those reasonable $\Lambda$ parameters. The agreement between experimental $P_{c}(4457)$ and our $4457 \mathrm{MeV}$ solutions also supports the judgment in turn.

However, it is not the end of our story. Let us turn to that $4457 \mathrm{MeV}$ solution with $J^{P}=\frac{1}{2}^{-}$. Differed from other molecular calculations, our approaches indicate this additional state in the $I=\frac{1}{2} \Sigma_{c} \bar{D}^{*}$ channel. This $P_{c}$ state is an excitation of $P_{c}(4440)$ which carries $J^{P}=\frac{1}{2}{ }^{-}$. The most interesting thing is that it has mass $M=4.456 \mathrm{GeV}$, which is located right at the $P_{c}(4457)$ mass region. Therefore we speculate that the $P_{c}(4457)$ signal discovered by LHCb may contain two overlapped signals: spin $-\frac{1}{2}$ and spin $-\frac{3}{2}$.

We now refer to the spin $-\frac{1}{2}$ signal as $P_{c}^{\prime}(4457)$. In a word, we totally determine four $\Sigma_{c} \bar{D}^{(*)}$ molecular states: $P_{c}$ (4312), $P_{c}(4440), P_{c}(4457)$, and $P_{c}^{\prime}(4457)$, where the last two are mixed as the observed signal in the $J / \psi p$ mass spectrum [23].

The BS wave functions of $P_{c}(4312), P_{c}(4440)$, $P_{c}(4457)$, and $P_{c}^{\prime}(4457)$ are displayed in Fig. 3. We can see that $f_{2}$ is dominant in $P_{c}(4312)$ 's wave function, while $g_{2}$ is quite prominent in the wave functions of $P_{c}(4440)$ and $P_{c}^{\prime}$ (4457). In general, we observe that the wave functions of the $P_{c}$ states are mixtures of $S, P, D$ waves and even radial exited components. Notice that in our framework, there only exists a limited number (four in our case) of bound states.

$P_{c}^{\prime}(4457)$ predicted in our work is mainly a first radial excitation, which means it has a similar property comparing to $P_{c}(4440)$. Furthermore the mass gap between $P_{c}(4440)$ and $P_{c}^{\prime}(4457)$ are just $\sim 17 \mathrm{MeV}$; we believe their widths are quite close. However, as a radial excitation, we prefer a smaller production ratio of $P_{c}^{\prime}(4457)$; therefore it is reasonable that $\mathrm{LHCb}$ can describe the $4457 \mathrm{MeV}$ peak now without additional $P_{c}^{\prime}(4457)$.

Indeed, with the limited information in [23] we can not trace $P_{c}^{\prime}(4457)$ for now. We expect that LHCb can further investigate quantum numbers and more decay channels, as well as add $P_{c}^{\prime}(4457)$ in their amplitude analysis, to justify our predictions. For example, LHCb can include only spin$\frac{1}{2}$ or both spin- $\frac{3}{2}$ and spin- $\frac{1}{2}$ at $4457 \mathrm{Mev}$ for comparison. On the other hand, for providing more useful and specific information, we will theoretically investigate the decay properties and production mechanism in future work.

In addition, we did not find any $I=\frac{3}{2}$ partners of the $\Sigma_{c} \bar{D}^{(*)}$ systems.

Notice that, in our calculations we did not consider the influences of other channels such as $\Sigma_{c}^{*} \bar{D}^{(*)}, \Lambda_{c} \bar{D}^{*}$ and $p J / \psi$. Although they would not change the main conclusions according to the calculations in Refs. [24,26,73], they are still needed for better descriptions of the $P_{c}$ states. Furthermore, they may be important for other properties such as decay rates. Therefore we will study the coupled channel formalism in a forthcoming paper. 


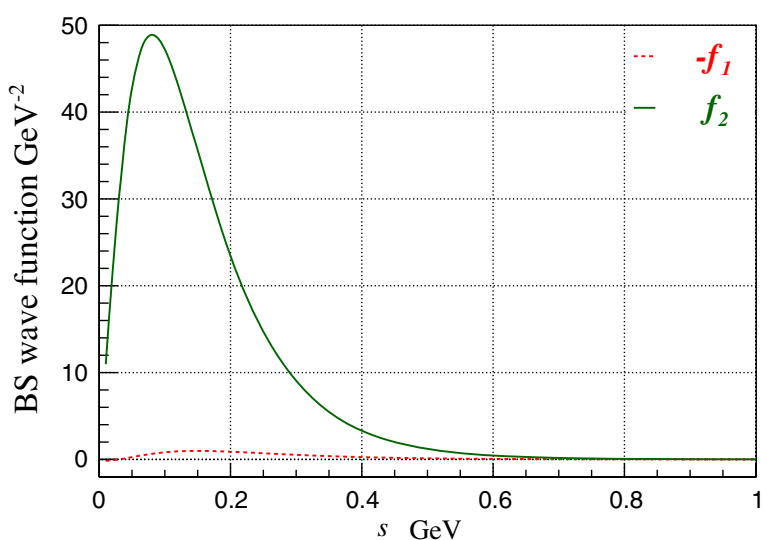

(a) $P_{c}(4312)$

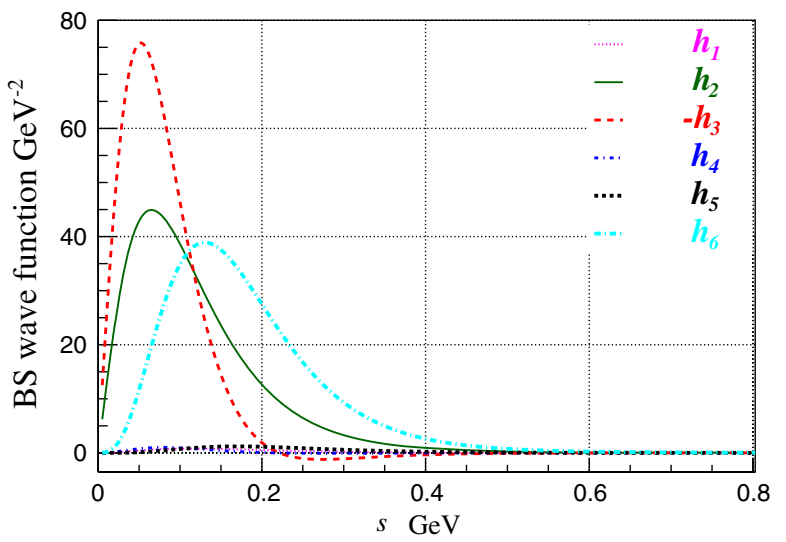

(c) $P_{c}(4457)$

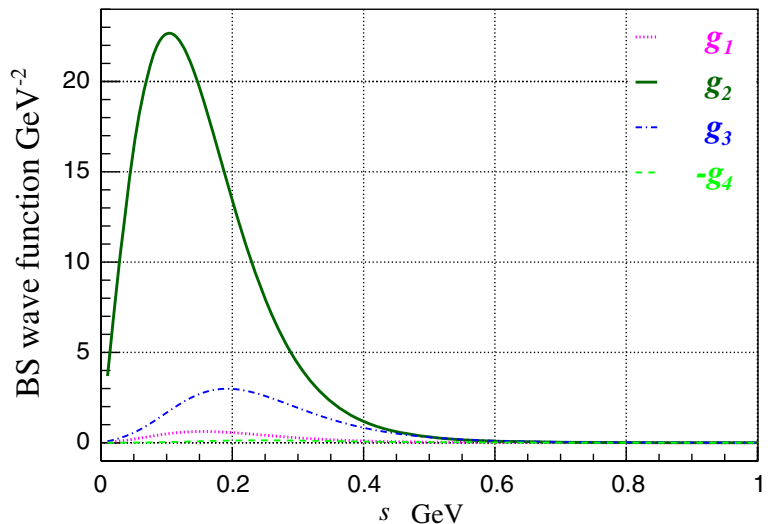

(b) $P_{c}(4440)$

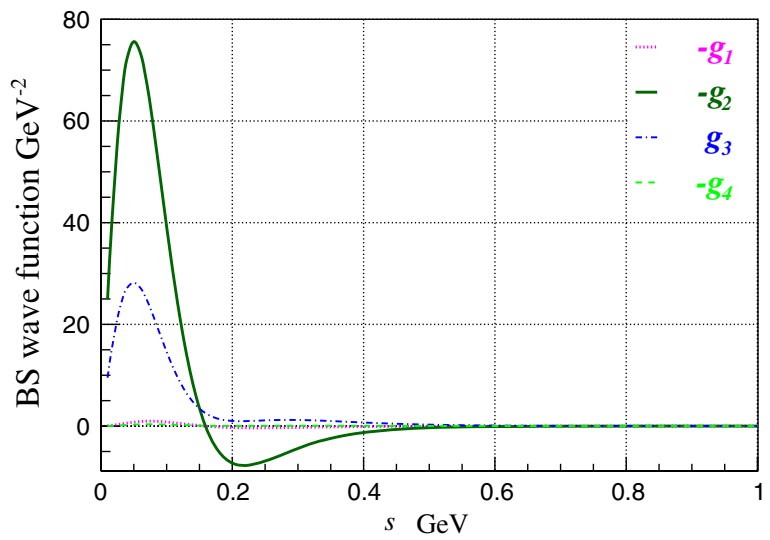

(d) $P_{c}^{\prime}(4457)$

FIG. 3. The BS radial wave functions of (a) $P_{c}(4312)$, (b) $P_{c}(4440)$, (c) $P_{c}(4457)$, and (d) $P_{c}^{\prime}(4457)$.

\section{SUMMARY}

Recently, LHCb reexamined the process $\Lambda_{b} \rightarrow J / \psi K^{-} p$, and discovered three $P_{c}$ pentaquarks: $P_{c}(4312), P_{c}(4440)$, and $P_{c}(4457)$ [23]. Although their molecular nature has been confirmed by many papers, we still think there may emerge interesting structures among three $P_{c}$ signals considering the experience that old $P_{c}(4450)$ splits into $P_{c}(4440)$ and $P_{c}(4457)$.

Benefited from the effective Lagrangians respecting the chiral and heavy quark symmetry, as well as the instantaneous Bethe-Salpeter equation, we investigate $\Sigma_{c} \bar{D}^{(*)}$ interactions and observed $P_{c}$ signals. First, we calculate the $\Sigma_{c} \bar{D}^{(*)}$ interaction amplitudes according to the effective Lagrangians, and study the behaviors of the potentials (Fig. 2). Then, we study the BS formalism of the $\Sigma_{c} \bar{D}^{(*)}$ system, and iterate former interactions into the BS equations. Finally, we obtain molecular solutions as well as their BS wave functions (Fig. 3).

Our calculations show that, $P_{c}(4312)$ can be treated as an $I=\frac{1}{2}-\Sigma_{c} \bar{D}$ molecular state with $J^{P}=\frac{1}{2}^{-}$, while $P_{c}(4440)$ is a good candidate of an $I=\frac{1}{2} \Sigma_{c} \bar{D}^{*}$ molecule, which also carries $J^{P}=\frac{1}{2}^{-}$.
Differed from other molecular calculations, our work indeed indicates not one but two bound states in the $P_{c}(4457)$ mass region: one is $\Sigma_{c} \bar{D}^{*}$ with $J^{P}=\frac{3}{2}$, another is $\Sigma_{c} \bar{D}^{*}$ carrying $J^{P}=\frac{1}{2}$, which is just an excitation of $P_{c}(4440)$. Therefore we conclude that the $P_{c}(4457)$ signal discovered by LHCb might be a mixture of $J^{P}=\frac{1}{2}^{-}$and $\frac{3-}{2}$ states.

In a word, we totally determine four molecular states $P_{c}$ (4312), $P_{c}(4440), P_{c}(4457)$, and $P_{c}^{\prime}(4457)$, which matches to three LHCb signals. We speculate the existence of the additional excitation $\left[P_{c}^{\prime}(4457)\right]$ is necessary, because the relatively large excitation space of ground $P_{c}(4440)$. Also, $P_{c}^{\prime}(4457)$ has a very similar decay property with $P_{c}(4440)$.

Moreover, we did not support any existences of corresponding isospin- $-\frac{3}{2}$ molecular solutions in our calculations.

We expect $\mathrm{LHCb}$ can perform amplitude analysis with more data samples, as well as search for other decay channels, to separate two states in the $4457 \mathrm{MeV}$ signal region. We hope our conclusions can be testified in the future.

\section{ACKNOWLEDGMENTS}

We thank Xiang Liu for helpful discussions. This work is supported by the Fundamental Research Funds for the 
Central Universities, under Grants No. 31020180QD118 and No. 310201911QD054. This work is supported in part by the National Natural Science Foundation of China (NSFC) under Grants No. 11575048, No. 11745006, No. 11535002, No. 11675239, and No. 11821505.

\section{APPENDIX: SOME EXPRESSIONS FOR DERIVATIONS OF THE SALPETER EQUATION}

To perform the contour integral, we rewrite the propagators:

$S\left(p_{2}\right)=i \frac{1}{\not \not \supset 2_{2}-m_{2}}=-i\left(\frac{\Lambda^{+}}{q_{P}-\zeta_{2}^{+}-i \epsilon}+\frac{\Lambda^{-}}{q_{P}-\zeta_{2}^{-}+i \epsilon}\right)$,

$D\left(p_{1}\right)=i \frac{1}{p_{1}^{2}-m_{1}^{2}}=i \frac{1}{2 \omega_{1}}\left(\frac{1}{q_{P}-\zeta_{1}^{+}+i \epsilon}-\frac{1}{q_{P}-\zeta_{1}^{-}-i \epsilon}\right)$,

where $\zeta_{2}^{ \pm}=\alpha_{2} M \mp \omega_{2}, \zeta_{1}^{+}=-\alpha_{1} M \pm \omega_{1}$.
Inserting the above expressions into Eq. (28), then performing the contour integral over $q_{P}$, we obtain the three-dimensional Salpeter equation (30). Utilizing $\varphi_{\alpha}^{ \pm}$ defined in Eq. (32), the equation further reduces two coupled equations:

$$
\left(M-\omega_{1}-\omega_{2}\right) \varphi_{\alpha}^{+}=\frac{\Lambda^{+} d_{\alpha \beta} \Gamma^{\beta}}{2 \omega_{1}},
$$

$$
\left(M+\omega_{1}+\omega_{2}\right) \varphi_{\alpha}^{-}=\frac{\Lambda^{-} d_{\alpha \beta} \Gamma^{\beta}}{2 \omega_{1}},
$$

which can otherwise be simplified to Eq. (33).

The BS vertex can also be expressed by the Salpeter wave function as

$$
\Gamma^{\alpha}(P, q)=S^{-1}\left(p_{2}\right) D^{-1}\left(p_{1}\right) \vartheta^{\alpha \beta} \psi_{\beta}(P, q) .
$$

[1] H. X. Chen, W. Chen, X. Liu, and S. L. Zhu, Phys. Rep. 639, 1 (2016).

[2] F. K. Guo, C. Hanhart, U. G. Meißner, Q. Wang, Q. Zhao, and B. S. Zou, Rev. Mod. Phys. 90, 015004 (2018).

[3] Y. R. Liu, H. X. Chen, W. Chen, X. Liu, and S. L. Zhu, Prog. Part. Nucl. Phys. 107, 237 (2019).

[4] N. Brambilla, S. Eidelman, C. Hanhart, A. Nefediev, C. P. Shen, C. E. Thomas, A. Vairo, and C. Z. Yuan, arXiv: 1907.07583.

[5] R. Aaij et al. (LHCb Collaboration), Phys. Rev. Lett. 115, 072001 (2015).

[6] R. Chen, X. Liu, X. Q. Li, and S. L. Zhu, Phys. Rev. Lett. 115, 132002 (2015).

[7] H. X. Chen, W. Chen, X. Liu, T. G. Steele, and S. L. Zhu, Phys. Rev. Lett. 115, 172001 (2015).

[8] M. Karliner and J. L. Rosner, Phys. Rev. Lett. 115, 122001 (2015).

[9] K. Azizi, Y. Sarac, and H. Sundu, Phys. Rev. D 95, 094016 (2017).

[10] K. Azizi, Y. Sarac, and H. Sundu, Phys. Lett. B 782, 694 (2018).

[11] L. Maiani, A. D. Polosa, and V. Riquer, Phys. Lett. B 749, 289 (2015).

[12] V. V. Anisovich, M. A. Matveev, J. Nyiri, A. V. Sarantsev, and A. N. Semenova, arXiv:1507.07652.

[13] R. F. Lebed, Phys. Lett. B 749, 454 (2015).

[14] Z. G. Wang and T. Huang, Eur. Phys. J. C 76, 43 (2016).

[15] F. K. Guo, U. G. Meißner, W. Wang, and Z. Yang, Phys. Rev. D 92, 071502 (2015).

[16] U. G. Meißner and J. A. Oller, Phys. Lett. B 751, 59 (2015).
[17] X. H. Liu, Q. Wang, and Q. Zhao, Phys. Lett. B 757, 231 (2016).

[18] J. J. Wu, R. Molina, E. Oset, and B. S. Zou, Phys. Rev. Lett. 105, 232001 (2010).

[19] Z. C. Yang, Z. F. Sun, J. He, X. Liu, and S. L. Zhu, Chin. Phys. C 36, 6 (2012).

[20] W. L. Wang, F. Huang, Z. Y. Zhang, and B. S. Zou, Phys. Rev. C 84, 015203 (2011).

[21] J. J. Wu, T.-S. H. Lee, and B. S. Zou, Phys. Rev. C 85, 044002 (2012).

[22] X. Q. Li and X. Liu, Eur. Phys. J. C 74, 3198 (2014).

[23] R. Aaij et al. (LHCb Collaboration), Phys. Rev. Lett. 122, 222001 (2019).

[24] R. Chen, Z. F. Sun, X. Liu, and S. L. Zhu, Phys. Rev. D 100, 011502 (2019).

[25] M. Z. Liu, Y. W. Pan, F. Z. Peng, M. S. Snchez, L. S. Geng, A. Hosaka, and M. P. Valderrama, Phys. Rev. Lett. 122, 242001 (2019).

[26] J. He, Eur. Phys. J. C 79, 393 (2019).

[27] C. W. Xiao, J. Nieves, and E. Oset, Phys. Rev. D 100, 014021 (2019).

[28] L. Meng, B. Wang, G. J. Wang, and S. L. Zhu, Phys. Rev. D 100, 014031 (2019).

[29] Y. Yamaguchi, H. Garca-Tecocoatzi, A. Giachino, A. Hosaka, E. Santopinto, S. Takeuchi, and M. Takizawa, arXiv:1907.04684.

[30] M. P. Valderrama, Phys. Rev. D 100, 094028 (2019).

[31] M. Z. Liu, T. W. Wu, M. S. Snchez, M. P. Valderrama, L. S. Geng, and J. J. Xie, arXiv:1907.06093.

[32] H. Huang, J. He, and J. Ping, arXiv:1904.00221. 
[33] J. J. Wu, T.-S. H. Lee, and B. S. Zou, Phys. Rev. C 100, 035206 (2019).

[34] S. Sakai, H. J. Jing, and F. K. Guo, Phys. Rev. D 100, 074007 (2019).

[35] Z. H. Guo and J. A. Oller, Phys. Lett. B 793, 144 (2019).

[36] C. J. Xiao, Y. Huang, Y. B. Dong, L. S. Geng, and D. Y. Chen, Phys. Rev. D 100, 014022 (2019).

[37] H. X. Chen, W. Chen, and S. L. Zhu, Phys. Rev. D 100, 051501 (2019).

[38] M. B. Voloshin, Phys. Rev. D 100, 034020 (2019).

[39] F. K. Guo, H. J. Jing, U. G. Meißner, and S. Sakai, Phys. Rev. D 99, 091501 (2019).

[40] Y. H. Lin and B. S. Zou, Phys. Rev. D 100, 056005 (2019).

[41] T. Gutsche and V.E. Lyubovitskij, Phys. Rev. D 100, 094031 (2019).

[42] T. J. Burns and E. S. Swanson, Phys. Rev. D 100, 114033 (2019).

[43] Z. G. Wang and X. Wang, arXiv:1907.04582.

[44] M. L. Du, V. Baru, F. K. Guo, C. Hanhart, U. G. Meißner, J. A. Oller, and Q. Wang, Phys. Rev. Lett. 124, 072001 (2020).

[45] B. Wang, L. Meng, and S. L. Zhu, J. High Energy Phys. 11 (2019) 108.

[46] X. Z. Weng, X. L. Chen, W. Z. Deng, and S. L. Zhu, Phys. Rev. D 100, 016014 (2019).

[47] A. Ali and A. Y. Parkhomenko, Phys. Lett. B 793, 365 (2019).

[48] A. Ali, I. Ahmed, M. J. Aslam, A. Y. Parkhomenko, and A. Rehman, J. High Energy Phys. 10 (2019) 256.

[49] Z. G. Wang, Int. J. Mod. Phys. A 35, 2050003 (2020).

[50] J. F. Giron, R. F. Lebed, and C. T. Peterson, J. High Energy Phys. 05 (2019) 061.

[51] J. B. Cheng and Y. R. Liu, Phys. Rev. D 100, 054002 (2019).

[52] F. Stancu, Eur. Phys. J. C 79, 957 (2019).

[53] M. I. Eides, V. Y. Petrov, and M. V. Polyakov, arXiv: 1904.11616.

[54] G. Burdman and J. F. Donoghue, Phys. Lett. B 280, 287 (1992).

[55] M. B. Wise, Phys. Rev. D 45, R2188 (1992).
[56] T. M. Yan, H. Y. Cheng, C. Y. Cheung, G. L. Lin, Y. C. Lin, and H. L. Yu, Phys. Rev. D 46, 1148 (1992); 55, 5851(E) (1997).

[57] R. Casalbuoni, A. Deandrea, N. Di Bartolomeo, R. Gatto, F. Feruglio, and G. Nardulli, Phys. Rep. 281, 145 (1997).

[58] H. Y. Cheng and C. K. Chua, Phys. Rev. D 75, 014006 (2007).

[59] C. H. Chang, J. K. Chen, X. Q. Li, and G. L. Wang, Commun. Theor. Phys. 43, 113 (2005).

[60] C. H. Chang and G. L. Wang, Sci. China Phys. Mech. Astron. 53, 2005 (2010).

[61] C. H. Chang, C. S. Kim, and G. L. Wang, Phys. Lett. B 623, 218 (2005).

[62] Z. H. Wang, G. L. Wang, and C. H. Chang, J. Phys. G 39, 015009 (2012).

[63] T. Wang, G. L. Wang, H. F. Fu, and W. L. Ju, J. High Energy Phys. 07 (2013) 120.

[64] T. Wang, G. L. Wang, W. L. Ju, and Y. Jiang, J. High Energy Phys. 03 (2013) 110.

[65] Q. Li, T. Wang, Y. Jiang, H. Yuan, and G. L. Wang, Eur. Phys. J. C 76, 454 (2016).

[66] Q. Li, T. Wang, Y. Jiang, H. Yuan, T. Zhou, and G. L. Wang, Eur. Phys. J. C 77, 12 (2017).

[67] Q. Li, Y. Jiang, T. Wang, H. Yuan, G. L. Wang, and C. H. Chang, Eur. Phys. J. C 77, 297 (2017).

[68] Q. Li, C.-H. Chang, S.-X. Qin, and G.-L. Wang, Chin. Phys. C 44, 013102 (2020).

[69] R. Machleidt and D. R. Entem, Phys. Rep. 503, 1 (2011).

[70] E. Epelbaum, H. Krebs, and P. Reinert, arXiv:1911.11875.

[71] X. L. Ren, K. W. Li, L. S. Geng, B. W. Long, P. Ring, and J. Meng, Chin. Phys. C 42, 014103 (2018).

[72] H. Xu, B. Wang, Z. W. Liu, and X. Liu, Phys. Rev. D 99, 014027 (2019).

[73] J. He and D. Y. Chen, Eur. Phys. J. C 79, 887 (2019).

[74] H. W. Ke, M. Li, X. H. Liu, and X. Q. Li, Phys. Rev. D 101, 014024 (2020).

[75] R. E. Behrends and C. Fronsdal, Phys. Rev. 106, 345 (1957).

[76] F. L. Wang, R. Chen, Z. W. Liu, and X. Liu, Phys. Rev. C 101, 025201 (2020). 